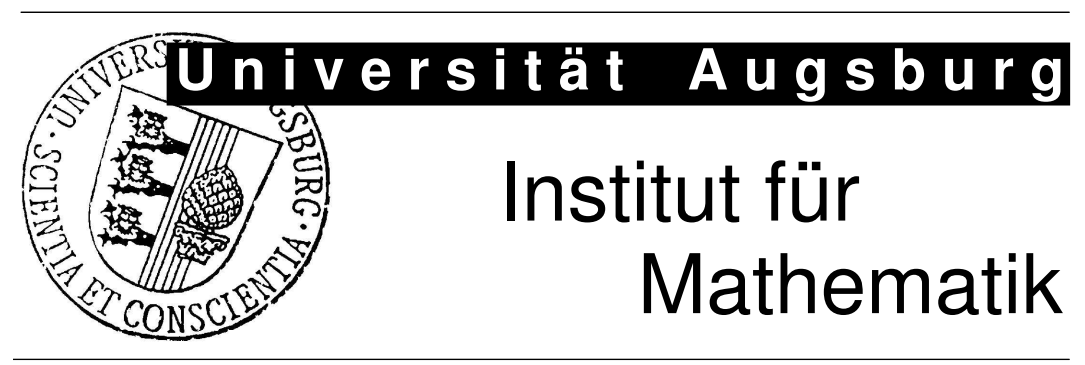

Ronald H.W. Hoppe; Tobias Lipp

Optimal Control of European Double Barrier Basket Options

Preprint Nr. 04/2011 - 16. März 2011

Institut für Mathematik, Universitätsstraße, D-86135 Augsburg

http://www.math.uni-augsburg.de/ 


\section{Impressum:}

\section{Herausgeber:}

Institut für Mathematik

Universität Augsburg

86135 Augsburg

http://www.math.uni-augsburg.de/pages/de/forschung/preprints.shtml

\section{ViSdP:}

Ronald H.W. Hoppe

Institut für Mathematik

Universität Augsburg

86135 Augsburg

Preprint: Sämtliche Rechte verbleiben den Autoren (C) 2011 


\title{
Optimal control of European double barrier basket options
}

\author{
R. H.W. HOPPE ${ }^{* \dagger}$ and T. LIPP $\$ \S$
}

4th March 2011

\begin{abstract}
We consider European double barrier basket call options on two underlyings with an upper and a lower knock-out barrier featuring a finite number of cash settlements at prespecified values of the underlyings between the strike and the upper barrier. The bilaterally constrained cash settlements are considered as controls that have to be chosen such that the Delta of the option is as close as possible to a predefined constant profit/loss. This leads to a control constrained optimal control problem for the two-dimensional Black-Scholes equation with Dirichlet boundary control and finite time control. Based on the variational formulation of the problem in an appropriate Sobolev space setting, we prove the existence of a unique solution and state the first order necessary optimality conditions. A semi-discretization in space by conforming P1 finite elements with respect to a simplicial triangulation of the computational domain gives rise to a semi-discrete control constrained optimal control problem for a linear system of first order ordinary differential equations. A further discretization in time by the backward Euler scheme results in a fully discrete optimization problem that is solved numerically by the projected gradient method with Armijo line search. Numerical examples for some selected test cases illustrate the benefits of hedging with European double barrier basket options in case of optimally controlled cash settlements.
\end{abstract}

Keywords: European double barrier basket options, multiple cash settlements, optimal control, Dirichlet and final time control, Black-Scholes equation, finite element discretization

\section{Introduction}

Options that are different from plain vanilla American or European call or put options are commonly referred to as exotic options (cf., e.g., [16,24,34]). Among the exotic options, those of single or double barrier type are of particular interest. Such options are either activated (knock in options) or expire (knock out options) when the value of the underlying reaches some predetermined upper and/or lower bound (barrier). The valuation of a single barrier option one one underlying has already

\footnotetext{
* Dept. of Math., University of Houston, Houston TX 77204-3008, U.S.A.

$\dagger$ Inst. of Math., University of Augsburg, D-86159 Augsburg, Germany

†Laboratoire Jacques-Louis Lions, Université Paris VI, F-75013 Paris, France

$\S_{\text {Inst. of Math., University of Augsburg, D-86159 Augsburg, Germany }}$

The first author has been supported by by the NSF under Grants DMS-0707602, DMS-0810176, DMS-0811153, and DMS-0914788, by the DFG within the Priority Program SPP 1253, by the BMBF within the projects 'FROPT' and 'MeFreSim', and by the ESF within the Research Networking Programme 'OPTPDE'.
} 
been dealt with in the seminal paper by Merton [22] and subsequently studied in $[6,9,27,29]$. The first contribution addressing barrier options with more than one underlying is [15]. In particular, the authors are concerned with barrier options on a single stock with the barrier being determined by another asset, whereas valuation formulas for barrier options on a basket have been derived later in $[18,33]$. Hedging techniques for barrier options have been considered by different approaches including static hedging based on the equivalence to contingent claims with specifically adjusted pay-off functions $[7,8,26]$, the partial differential equation (PDE) formulation $[2,11,19,23,26,30]$, and stochastic optimization [14,20,21].

In this paper, we will follow an optimal control approach for hedging barrier options with multiple cash settlements at the option's expiration [4]. The paper is organized as follows: In section 2, we consider hedging with European double barrier basket call options on two underlyings featuring a finite number of cash settlements at predetermined values of the underlyings between the strike and the upper barrier. The cash settlements are treated as bilaterally constrained control variables that have to be chosen in such a way that a tracking type objective functional in terms of the Delta of the option is minimized. This amounts to the solution of a control constrained optimal control problem for the Black-Scholes equation in some space-time domain $Q:=\Omega \times(0, T), T>0$, where $\Omega$ is a trapezoidal domain in $\mathbb{R}^{2}$ determined by the lower and upper barriers $K_{\min }$ and $K_{\max }$. The cash settlement at the upper barrier occurs as a Dirichlet boundary control, whereas the remaining cash settlements enter as a final time control vector. A particular feature is that the Dirichlet boundary conditions on the boundaries parallel to the coordinate axes are given by the solution of associated 1D Black-Scholes equations. In section 3, we reformulate the problem as an initial control/Dirichlet boundary control problem by means of a simple transformation in time and deal with its weak formulation in a weighted Sobolev space setting. Section 4 is devoted to the derivation of the first order necessary optimality conditions involving adjoint states that satisfy backward in time parabolic PDEs as well as a variational inequality due to the bilateral constraints on the control. In section 5, we are concerned with a semi-discretization in space by conforming P1 finite elements with respect to a simplicial triangulation of the computational domain. The semi-discrete control problem amounts to the minimization of a semi-discrete objective functional subject to systems of first order ordinary differential equations (ODEs) obtained by the finite element approximation in space and the constraints imposed on the controls. It thus represents a control constrained initial control problem for the respective systems of first order ODEs in terms of the associated mass and stiffness matrices as well as input matrices expressing the input from the semi-discretized boundary controls at the upper barrier. The corresponding semi-discrete optimality system nicely reflects the intrinsic couplings between the states, their adjoint counterparts, and the controls. Using a further discretization in time by the implicit Euler method, the resulting fully discrete optimality system is studied in section 6. Its numerical solution is realized by the projected BFGS method. Finally, section 7 contains a documentation of numerical results illustrating the application of the optimal control approach. 


\section{Hedging with European double barrier basket options}

We consider a European double barrier basket call option on a basket consisting of two assets with prices $S_{i}, 1 \leqslant i \leqslant 2$. We assume that the option has maturity $T>0$, strike $K>0$, and barriers $K_{\min }, K_{\max }$ satisfying $K_{\min }<K<K_{\max }$. Hence, the spatial domain $\Omega \subset \mathbb{R}_{+}^{2}$ for the price $y(S, t), S=\left(S_{1}, S_{2}\right) \in \Omega, t \in[0, T]$, of the option is given by the trapezoid

$$
\Omega:=\left\{S=\left(S_{1}, S_{2}\right) \in \mathbb{R}_{+}^{2}\left|K_{\text {min }}<\right| S \mid<K_{\max }\right\},
$$

where $|S|:=S_{1}+S_{2}$, with boundaries

$$
\begin{array}{rll}
\Gamma_{1}:=\left(K_{\min }, K_{\max }\right) \times\{0\} & , & \Gamma_{2}:=\{0\} \times\left(K_{\min }, K_{\max }\right), \\
\Gamma_{3}:=\left\{S \in \mathbb{R}_{+}^{2}|| S \mid=K_{\min }\right\} & , \quad \Gamma_{4}:=\left\{S \in \mathbb{R}_{+}^{2}|| S \mid=K_{\max }\right\} .
\end{array}
$$

We denote by $r=r(t), t \in[0, T]$, the risk-free interest rate and by $\sigma_{k}=\sigma_{k}(S, t), 1 \leqslant$ $k \leqslant 2, S \in \Omega, t \in[0, T]$, the volatilities of the assets. Moreover, we refer to $\rho=$ $\left(\rho_{k \ell}\right)_{k, \ell=1}^{2}$ with $\rho_{k k}=1,1 \leqslant k \leqslant 2$, and $\rho_{12}=\rho_{21}=2 \rho /\left(1+\rho^{2}\right),-1<\rho<+1$, as the correlation matrix. We set $\xi=\left(\xi_{k \ell}\right)_{k, \ell=1}^{2}$ where $\xi_{k \ell}:=\rho_{k \ell} \sigma_{k} \sigma_{\ell}, 1 \leqslant k, \ell \leqslant 2$. Then, it is well-known (cf., e.g., [1,4,31]) that the price $y_{Q}, Q:=\Omega \times(0, T)$, of the option satisfies the following boundary value problem for the Black-Scholes equation with a final time condition at maturity $T$ :

$$
\begin{aligned}
\frac{\partial y_{Q}}{\partial t}+L_{\Omega}(t) y_{Q} & =0 \quad \text { in } Q:=\Omega \times(0, T), \\
y_{Q} & =y_{\Sigma_{j}} \quad \text { on } \Sigma_{j}:=\Gamma_{j} \times(0, T), 1 \leqslant j \leqslant 4, \\
y_{Q}(\cdot, T) & =y_{Q, T} \quad \text { in } \Omega .
\end{aligned}
$$

Here, $L_{\Omega}(t), t \in[0, T]$, stands for the second order elliptic operator

$$
L_{\Omega}(t):=\frac{1}{2} \sum_{k, \ell=1}^{2} \xi_{k \ell} S_{k} S_{\ell} \frac{\partial^{2}}{\partial S_{k} \partial S_{\ell}}+r \sum_{k=1}^{2} S_{k} \frac{\partial}{\partial S_{k}}-r .
$$

The payoff $y_{Q, T}$ at maturity $T$ is given by

$$
y_{Q, T}(S):=(|S|-K)_{+}, S \in \Omega .
$$

Moreover, $y_{\Sigma_{3}}=0$ and the constant $y_{\Sigma_{4}}$ represents a cash settlement at the upper barrier $\Sigma_{4}$, whereas $y_{\Sigma_{v}}, 1 \leqslant v \leqslant 2$, have to be computed as the solutions of the one-dimensional Black-Scholes equations

$$
\begin{aligned}
\frac{\partial y_{\Sigma_{v}}}{\partial t}+L_{\Gamma_{v}}(t) y_{\Sigma_{v}} & =0 \quad \text { in } \Sigma_{v}:=\Gamma_{v} \times(0, T), \\
y_{\Sigma_{v}}\left(S_{v}, t\right) & =\left\{\begin{array}{c}
0, S_{v}=K_{\min } \\
y_{4}, S_{v}=K_{\max }
\end{array}, t \in(0, T),\right. \\
y_{\Sigma_{v}}(\cdot, T) & =\left.y_{Q, T}\right|_{\Gamma_{v}} \quad \text { in } \Gamma_{v},
\end{aligned}
$$


where $L_{\Gamma_{v}}(t), 1 \leqslant v \leqslant 2, t \in[0, T]$, are the second order elliptic operators

$$
L_{\Gamma_{v}}(t):=\frac{1}{2} \sigma_{v}^{2} S_{v}^{2} \frac{\partial^{2}}{\partial S_{v}^{2}}+r S_{v} \frac{\partial}{\partial S_{v}}-r
$$

However, for hedging with European double barrier basket options, more complicated payoffs are possible. In fact, given a partitioning

$$
K=: K_{0}<K_{1}<\cdots<K_{M}:=K_{\max }, M \in \mathbb{N},
$$

of $\left[K, K_{\max }\right]$ with $K_{i}:=K+i \delta_{|S|}, 0 \leqslant i \leqslant M, \delta_{|S|}:=\left(K_{\max }-K\right) / M$ and setting formally $u:=\left(u_{1}, \cdots, u_{M}\right)^{T} \in \mathbb{R}_{+}^{M}$, as well as $K_{-1}=K_{\min }, u_{-1}=u_{0}=0$, we may choose $y_{4}=u_{M}$ and $y_{T}=g(u)$ where

$$
\begin{aligned}
& (g(u))(S)=u_{i-1} g_{1}^{(i)}(S)+u_{i} g_{2}^{(i)}(S) \text { for }|S| \in\left[K_{i-1}, K_{i}\right], i=0, \cdots, M, \\
& g_{1}^{(i)}(S):=\left(K_{i}-S\right) / \delta_{|S|}, g_{2}^{(i)}(S):=\left(S-K_{i-1}\right) / \delta_{|S|} .
\end{aligned}
$$

We may consider $u$ as a control vector that has to be chosen such that the Greek $\Delta:=\nabla y$ per asset point is as close to a prespecified profit $d=\left(d_{1}, d_{2}\right)^{T}$ as possible. The controls are subject to the constraints

$$
\begin{aligned}
u \in U_{a d} & :=\left\{v=\left(v_{1}, \cdots, v_{M}\right)^{T} \in \mathbb{R}_{+}^{M} \mid v_{i} \in U_{a d}^{(i)}, 1 \leqslant i \leqslant M\right\}, \\
U_{a d}^{(i)} & :=\left\{v_{i} \in \mathbb{R}_{+} \mid u_{i, \text { min }} \leqslant v_{i} \leqslant u_{i, \max }\right\} .
\end{aligned}
$$

We consider the following optimal control problem for the two-dimensional BlackScholes equation: Find $\left(y_{Q}, u\right)$ such that

$$
\inf _{y_{Q}, u} J\left(y_{Q}, u\right):=\frac{1}{2} \int_{0}^{T} \int_{\Omega}\left|\nabla y_{Q}-d\right|^{2} d S d t,
$$

subject to (2.3a)-(2.3c),(2.6a)-(2.6c), and (2.9).

\section{Variational formulation of the optimal control problem}

We use standard notation from Lebesgue and Sobolev space theory. In particular, given a bounded Lipschitz domain $\Omega \subset \mathbb{R}^{d}, d \in \mathbb{N}$, with boundary $\Gamma:=\partial \Omega$, for $D \subseteq \Omega$ we refer to $L^{p}(D), 1 \leqslant p \leqslant \infty$ as the Banach spaces of $p$-th power integrable functions $(p<\infty)$ and essentially bounded functions $(p=\infty)$ on $D$ with norm $\|\cdot\|_{L^{p}(D)}$. We denote by $L^{p}(D)_{+}$the positive cone in $L^{p}(D)$, i.e., $L^{p}(D)_{+}:=\left\{v \in L^{p}(D) \mid v \geqslant\right.$ 0 a.e. in $D$ \}. In case $p=2$, the space $L^{2}(D)$ is a Hilbert space whose inner product and norm will be referred to as $(\cdot, \cdot)_{L^{2}(D)}$. 
For $m \in \mathbb{N}_{0}$ and weight functions $\omega=\left(\omega_{\alpha}\right)_{|\alpha| \leqslant m}$ with $\omega_{\alpha} \in L^{\infty}(D)_{+}, \alpha=\left(\alpha_{1}, \cdots, \alpha_{d}\right) \in$ $\mathbb{N}_{0}^{d},|\alpha|:=\sum_{i=1}^{d} \alpha_{i}$, we denote by $W_{\omega}^{m, p}(D)$ the weighted Sobolev spaces with norms

$$
\|v\|_{W_{\omega}^{m, p}(D)}:=\left\{\begin{array}{c}
\left(\sum_{|\alpha| \leqslant m}\left\|\omega_{\alpha} D^{\alpha} v\right\|_{L^{p}(D)}^{p}\right)^{1 / p}, \text { if } p<\infty \\
\max _{|\alpha| \leqslant m}\left\|\omega_{\alpha} D^{\alpha} v\right\|_{L^{\infty}(D)}, \text { if } p=\infty
\end{array},\right.
$$

and refer to $|\cdot|_{W_{\omega}^{m, p}(D)}$ as the associated seminorms. In particular, for $|\alpha|=1$ we use the notation $\nabla \omega v:=\left(S_{1} \partial v / \partial S_{1}, \cdots, S_{d} \partial v / \partial S_{d}\right)^{T}$. For $p<\infty$ and $s \in \mathbb{R}_{+}, s=$ $m+\sigma, m \in \mathbb{N}_{0}, 0<\sigma<1$, we define the weighted Sobolev space $W_{\omega}^{s, p}(D)$ with norm $\|\cdot\|_{W_{\omega}^{s, p}(D)}$ in analogy to the standard, non-weighted case and refer to $W_{\omega, 0}^{s, p}(D)$ as the closure of $C_{0}^{\infty}(D)$ in $W_{\omega}^{s, p}(D)$. For $s<0$, we denote by $W_{\omega}^{-s, p}(D)$ the dual space of $W_{\omega, 0}^{-s, q}(D), p^{-1}+q^{-1}=1$. In case $p=2$, the spaces $W_{\omega}^{s, 2}(D)$ are Hilbert spaces. We will write $H_{\omega}^{s}(D)$ instead of $W_{\omega}^{s, 2}(D)$ and refer to $(\cdot, \cdot)_{H_{\omega}^{s}(D)}$ and $\|\cdot\|_{H_{\omega}^{s}(D)}$ as the inner products and associated norms. In the standard case $\omega_{\alpha} \equiv 1,|\alpha| \leqslant m$, we will drop the subindex $\omega$.

For a Banach space $X$ and its dual $X^{*}$, we refer to $\langle\cdot, \cdot\rangle_{X^{*}, X}$ as the dual pairing between $X^{*}$ and $X$. For Banach spaces $X_{i}, 1 \leqslant i \leqslant n, n \in \mathbb{N}$, and a function $v \in$ $\bigcap_{i=1}^{n} X_{i}$, we refer to $\|v\|_{\bigcap_{i=1}^{n} X_{i}}$ as the norm

$$
\|v\|_{\bigcap_{i=1}^{n} X_{i}}:=\max _{i \leqslant i \leqslant n}\|v\|_{X_{i}}
$$

Moreover, for $T>0$ and a Banach space $X$, we denote by $L^{p}((0, T), X), 1 \leqslant p \leqslant \infty$, and $C([0, T], X)$ the Banach spaces of functions $v:[0, T] \rightarrow X$ with norms

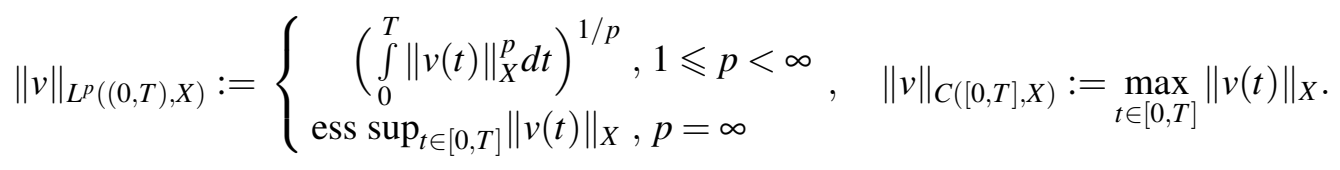

The spaces $W^{s, p}((0, T), X)$ and $H^{s}((0, T), X), s \in \mathbb{R}_{+}$, are defined likewise.

In particular, for a subspace $V \subset H_{\omega}^{1}(\Omega)$ with dual $V^{*}$ we will consider the space

$$
H^{1}\left((0, T), V^{*}\right) \cap L^{2}((0, T), V),
$$

and note that the following continuous embedding holds true

$$
H^{1}\left((0, T), V^{*}\right) \cap L^{2}((0, T), V) \subset C\left([0, T], L^{2}(\Omega)\right) .
$$

For $y \in H^{1}\left((0, T), V^{*}\right) \cap L^{2}((0, T), V)$, we further denote by $\gamma_{\Sigma^{\prime}}(y), \Sigma^{\prime} \subset \Sigma:=\Gamma \times$ $(0, T)$, the trace of $y$ on $\Sigma^{\prime}$.

In the sequel, $\Omega \subset \mathbb{R}_{+}^{2}$ will stand for the trapezoidal domain given by (2.1) and 
$\Gamma_{i} \subset \partial \Omega, 1 \leqslant i \leqslant 4$, for its boundaries as specified by (2.2). The weight functions $\omega_{\alpha}$ will be chosen according to

$$
w_{\alpha}=S_{\alpha_{1}} S_{\alpha_{2}}, \alpha=\left(\alpha_{1}, \alpha_{2}\right),|\alpha| \leqslant 2,
$$

with the convention that $S_{\alpha_{i}}=1$ for $\alpha_{i}=0,1 \leqslant i \leqslant 2$.

We reformulate the boundary and final time problems for the backward parabolic equations as initial-boundary value problems by means of the transformation $t \mapsto$ $T-t$. Keeping for notational simplicity the same notation for $y_{Q}$ and $y_{\Sigma_{v}}$ and the operators $L_{\Omega}, L_{\Sigma_{v}}, 1 \leqslant v \leqslant 2$, the initial-boundary value problems read as follows:

$$
\begin{gathered}
\frac{\partial y_{Q}}{\partial t}-L_{\Omega}(t) y_{Q}=0 \quad \text { in } Q:=\Omega \times(0, T), \\
y_{Q}=\left\{\begin{array}{r}
y_{v}, \text { on } \Sigma_{v}:=\Gamma_{v} \times(0, T), 1 \leqslant v \leqslant 2, \\
0, \text { on } \Sigma_{3}:=\Gamma_{3} \times(0, T) \\
u_{M}, \text { on } \Sigma_{4}:=\Gamma_{4} \times(0, T)
\end{array},\right. \\
y_{Q}(\cdot, 0)=g(u) \text { in } \Omega, \\
\frac{\partial y_{\Sigma_{v}}}{\partial t}-L_{\Gamma_{v}}(t) y_{\Sigma_{v}}=0 \quad \text { in } \Sigma_{v}, \\
y_{\Sigma_{v}}\left(S_{v}, t\right)=\left\{\begin{array}{c}
0, S_{v}=K_{\min } \\
u_{M}, S_{v}=K_{\max }
\end{array}, t \in(0, T),\right. \\
y_{\Sigma_{v}}(\cdot, 0)=\left.g(u)\right|_{\Gamma_{v}} \text { in } \Gamma_{v} .
\end{gathered}
$$

As far as the volatilities $\sigma_{k}, 1 \leqslant k \leqslant 2$, and the interest $r$ are concerned, we will impose the following assumptions:

(A1) The volatilities satisfy $\sigma_{k} \in C\left([0, T], C^{2}(\bar{\Omega})\right), 1 \leqslant k \leqslant 2$, and there exist constants $\sigma_{k}^{(\min )}>0, C_{\sigma_{k}}>0$, such that

$$
\begin{aligned}
& \sigma_{k}(S, t) \geqslant \sigma_{k}^{(\min )} \quad, \quad(S, t) \in \bar{Q}, 1 \leqslant k \leqslant 2, \\
& \left|S \cdot \nabla \sigma_{k}(S, t)\right| \leqslant C_{\sigma_{k}} \quad, \quad(S, t) \in \bar{Q}, 1 \leqslant k \leqslant 2 .
\end{aligned}
$$

(A2) The interest rate satisfies $r \in C([0, T])$ such that $r(t)>0, t \in[0, T]$.

For the correlation matrix $\xi=(\xi)_{k, \ell=1}^{2}$, it is an immediate consequence of assumption (A1) that $\xi_{k, \ell} \in C\left([0, T], C^{2}(\bar{\Omega})\right), 1 \leqslant k, \ell \leqslant 2$, and that there exists a constant $\xi_{\text {min }}>0$ such that for all $\eta \in \mathbb{R}^{2}$ there holds

$$
\sum_{k, \ell=1}^{2} \xi_{k, \ell}(S, t) \eta_{k} \eta_{\ell} \geqslant \xi_{\text {min }}|\eta|^{2} \quad, \quad(S, t) \in \bar{Q} .
$$


We now study the weak formulations of the initial-boundary value problems (3.5a)(3.5c) and (3.6a)-(3.6c):

A function $y_{Q} \in H^{1}\left((0, T), V^{*}\right) \cap L^{2}((0, T), V)$, where $V:=\left\{v \in H_{\omega}^{1}(\Omega)|v|_{\Sigma_{n u}}=\right.$ $\left.y_{\Sigma_{v}}, 1 \leqslant v \leqslant 2,\left.v\right|_{\Sigma_{3}}=0, v_{\Sigma_{4}}=u_{M}\right\}$, is called a weak solution of (3.5a)-(3.5c), if for all $v \in L^{2}\left((0, T), H_{\omega, 0}^{1}(\Omega)\right)$ there holds

$$
\begin{aligned}
\int_{0}^{T}\left\langle\frac{\partial y_{Q}}{\partial t}, v\right\rangle_{H_{\omega}^{-1}(\Omega), H_{\omega, 0}^{1}(\Omega)} d t+\int_{0}^{T} a\left(t ; y_{Q}, v\right) d t & =0, \\
y_{Q}(\cdot, 0) & =g(u)
\end{aligned}
$$

Here, the bilinear form $a(t ; \cdot, \cdot), t \in(0, T)$, is given by

$a(t ; y, v):=\int_{\Omega}\left(\frac{1}{2} \sum_{k, \ell=1}^{2} \xi_{k} S_{k} \frac{\partial y}{\partial S_{k}} S_{\ell} \frac{\partial v}{\partial S_{\ell}}-\sum_{k=1}^{2} r S_{k} \frac{\partial y}{\partial S_{k}} v-\left(\frac{1}{2} \sum_{k, \ell=1}^{2}\left(S_{k} S_{\ell} \frac{\partial \xi_{k \ell}}{\partial S_{\ell}}+\xi_{k \ell} S_{k}\right)-r\right) y v\right) d S$.

Likewise, a function $y_{\Sigma_{v}} \in H^{1}\left((0, T), V_{v}^{*}\right) \cap L^{2}\left((0, T), V_{v}\right)$, where $V_{v}:=\left\{v \in H_{\omega}^{1}\left(\Sigma_{v}\right) \mid v\left(K_{\text {min }}\right)=\right.$ $\left.0, v\left(K_{\max }\right)=u_{M}\right\}$, is said to be a weak solution of (3.6a)-(3.6c), if for all $v_{v} \in$ $L^{2}\left((0, T), H_{\omega, 0}^{1}\left(\Gamma_{v}\right)\right)$ there holds

$$
\begin{aligned}
\int_{0}^{T}\left\langle\frac{\partial y_{\Sigma_{v}}}{\partial t}, v_{v}\right\rangle d t+\int_{0}^{T} a_{v}\left(t ; y_{\Sigma_{v}}, v_{v}\right) d t & =0, \\
y_{\Sigma_{v}}(\cdot, 0) & =\left.g(u)\right|_{\Gamma_{v}} .
\end{aligned}
$$

Here, the bilinear form $a_{v}(t ; \cdot, \cdot), t \in(0, T)$, is given by

$$
a_{v}(t ; y, v):=\int_{\Gamma_{v}}\left(\frac{1}{2} \sigma_{v}^{2} S_{v} \frac{\partial y}{\partial S_{v}} S_{v} \frac{\partial v}{\partial S_{v}}-r S_{v} \frac{\partial y}{\partial S_{v}} v-\left(\sigma_{v}^{2} S_{v}+\sigma_{v} S_{v}^{2} \frac{\partial \sigma_{v}}{\partial S_{v}}-r\right) y v\right) d S_{v} .
$$

Theorem 3.1. For arbitrarily chosen but fixed $u \in \mathbb{R}^{M}$, the state equations (3.9a),(3.9b) and (3.10a),(3.10b) admit unique solutions $y_{Q} \in C([0, T], V) \cap L^{2}((0, T), V \cap$ $\left.H_{\omega}^{2}(\Omega)\right)$ and $y_{\Sigma_{v}} \in C\left([0, T], V_{v}\right) \cap L^{2}\left((0, T), V_{v} \cap H_{\omega}^{2}\left(\Gamma_{v}\right)\right), 1 \leqslant v \leqslant 2$. Moreover, for all $t \in(0, T)$ there holds

$$
\begin{gathered}
\exp (-2 \lambda t)\left\|y_{Q}(t)\right\|_{L^{2}(\Omega)}^{2}+2 \xi_{\min }^{2} \int_{0}^{t} \exp (-2 \lambda \tau)\left|y_{Q}(\tau)\right|_{V}^{2} d \tau \leqslant\|g(u)\|_{L^{2}(\Omega)}^{2}, \\
\exp \left(-2 \lambda_{v} t\right)\left\|y_{\Sigma_{v}}(t)\right\|_{L^{2}\left(\Gamma_{v}\right)}^{2}+\frac{1}{2}\left(\sigma_{v}^{(m i n)}\right)^{2} \int_{0}^{t} \exp \left(-2 \lambda_{v} \tau\right)\left|y_{\Sigma_{v}}(\tau)\right|_{V_{v}}^{2} d \tau \leqslant\|g(u)\|_{L^{2}\left(\Gamma_{v}\right)}^{2}, 1 \leqslant v \leqslant 2 .
\end{gathered}
$$


Proof. It follows from assumptions $\left(\mathbf{A}_{1}\right),\left(\mathbf{A}_{2}\right)$ and the Poincaré inequalities (cf., e.g., [1])

$$
\begin{aligned}
\|v\|_{L^{2}(\Omega)}^{2} & \leqslant 2 \int_{\Omega}\left|S \cdot \nabla_{\omega} v(S)\right|^{2} d S_{1} d S_{2}, v \in V, \\
\left\|v_{v}\right\|_{L^{2}\left(\Gamma_{v}\right)}^{2} & \leqslant 2 \int_{\Gamma_{v}}\left|S_{v} \frac{\partial v_{v}}{\partial S_{v}}\left(S_{v}\right)\right|^{2} d S_{v}, v_{v} \in V_{v}, 1 \leqslant v \leqslant 2,
\end{aligned}
$$

that the bilinear forms $a(t ; \cdot, \cdot)$ and $a_{v}(t ; \cdot, \cdot)$ satisfy Gârding-type inequalities uniformly in $t$, i.e., there exist constants $\lambda \geqslant 0$ and $\lambda_{v} \geqslant 0,1 \leqslant v \leqslant 2$, such that for all $t \in(0, T)$ there holds

$$
\begin{aligned}
a(t ; v, v) & \geqslant \frac{1}{4} \xi_{\text {min }}^{2}\|v\|_{V}^{2}-\lambda\|v\|_{L^{2}(\Omega)}^{2}, v \in V, \\
a_{v}(t ; v, v) & \geqslant \frac{1}{4}\left(\sigma_{v}^{(\min )}\right)^{2}\|v\|_{V_{v}}^{2}-\lambda_{v}\|v\|_{L^{2}\left(\Gamma_{v}\right)}^{2}, v \in V_{v}, 1 \leqslant v \leqslant 2 .
\end{aligned}
$$

Consequently, the initial-boundary value problems (3.9a),(3.9b) and (3.10a),(3.10b) have unique solutions $y_{Q} \in H^{1}\left((0, T), V^{*}\right) \cap L^{2}((0, T), V)$ and $y_{\Sigma_{v}} \in H^{1}\left((0, T), V_{v}^{*}\right) \cap$ $L^{2}\left((0, T) ; V_{v}\right), 1 \leqslant v \leqslant 2$, satisfying (3.11) (cf., e.g., Thm. 2.11 and section 2.6 in [1]). Moreover, standard regularity results for parabolic partial differential equations $[1,28]$ reveal $y_{Q} \in C([0, T], V) \cap L^{2}\left((0, T), V \cap H_{\omega}^{2}(\Omega)\right)$ and $y_{\Sigma_{v}} \in C\left([0, T], V_{v}\right) \cap$ $L^{2}\left((0, T), V_{v} \cap H_{\omega}^{2}\left(\Gamma_{v}\right)\right), 1 \leqslant v \leqslant 2$.

Based on the weak formulation of the state equations, the optimal control problem from section 2 reads as follows:

Find $(y, u)$, where $y=\left(y_{Q}, y_{\Sigma_{1}}, y_{\Sigma_{2}}\right), y_{Q} \in H^{1}\left((0, T), V^{*}\right) \cap L^{2}((0, T), V), y_{\Sigma_{v}} \in H^{1}\left((0, T), V_{v}^{*}\right) \cap$ $L^{2}\left((0, T) ; V_{v}\right), 1 \leqslant v \leqslant 2$, and $u \in U_{a d}$ such that

$$
\inf _{y, u} J(y, u):=\frac{1}{2} \int_{0}^{T} \int_{\Omega}\left|\nabla y_{Q}-d\right|^{2} d S d t,
$$

subject to (3.9a),(3.9b) and (3.10a),(3.10b).

Theorem 3.2. The optimal control problem (3.12a),(3.12b) admits a unique solution $(y, u)$.

Proof. We denote by $S: U_{a d} \rightarrow H^{1}\left((0, T), V^{*}\right) \cap L^{2}((0, T), V)$ and $S_{V}: U_{a d} \rightarrow$ $H^{1}\left((0, T), V_{v}^{*}\right) \cap L^{2}\left((0, T) ; V_{v}\right), 1 \leqslant v \leqslant 2$, the control-to-state maps which assign to 
an admissible control $u \in U_{a d}$ the unique solutions $y_{Q}$ and $y_{\Sigma_{v}}, 1 \leqslant v \leqslant 2$, of the state equations (3.9a),(3.9b) and (3.10a),(3.10b). Replacing $y=\left(y_{Q}, y_{\Sigma_{1}}, y_{\Sigma_{2}}\right)$ in (3.12a) with $\left(S(u), S_{1}(u), S_{2}(u)\right)$, the reduced formulation of (3.12a), (3.12b) is given by:

$$
\inf _{u \in U_{a d}} J^{r e d}(u):=\frac{1}{2} \int_{0}^{T} \int_{\Omega}|\nabla S(u)-d|^{2} d S d t,
$$

such that the triple $\left(S(u), S_{1}(u), S_{2}(u)\right)$ satisfies (3.9a),(3.9b) and (3.10a),(3.10b).

Let $\left(u_{n}\right)_{n \in \mathbb{N}}, u_{n} \in U_{a d}, n \in \mathbb{N}$, be a minimizing sequence. Due to the facts that $\left(u_{n}\right)_{n \in \mathbb{N}}$ is bounded and $U_{a d} \subset \mathbb{R}^{M}$ is closed, there exist a subsequence $\mathbb{N}^{\prime} \subset \mathbb{N}$ and $u^{*} \in U_{\text {ad }}$ such that $u_{n} \rightarrow u^{*}\left(\mathbb{N}^{\prime} \ni n \rightarrow \infty\right)$. From the continuity of the control-to-state maps we deduce

$$
S\left(u_{n}\right) \rightarrow S\left(u^{*}\right) \quad, \quad S_{v}\left(u_{n}\right) \rightarrow S_{v}\left(u^{*}\right) \quad\left(\mathbb{N}^{\prime} \ni n \rightarrow \infty\right) .
$$

Moreover, $\left(S\left(u^{*}\right), S_{1}\left(u^{*}\right), S_{2}\left(u^{*}\right)\right)$ satisfies (3.9a),(3.9b) and (3.10a),(3.10b). Taking additionally the continuity of $g$ into account, we find

$$
J^{r e d}\left(u_{n}\right) \rightarrow J^{r e d}\left(u^{*}\right) \quad\left(\mathbb{N}^{\prime} \ni n \rightarrow \infty\right),
$$

which allows to conclude.

\section{Necessary optimality conditions}

The first order necessary optimality conditions can be stated in terms of $(y, u), y=$ $\left(y_{Q}, y_{\Sigma_{1}}, y_{\Sigma_{2}}\right)$, and an adjoint state $p \in W_{0}(0, T)$ that is the solution of a final time problem on $Q$ with homogeneous Dirichlet boundary conditions.

Theorem 4.1. If $(y, u) \in W(0, T) \times U_{a d}$ is the optimal solution of (3.12a),(3.12b), there exists $p_{Q} \in W_{0}(0, T)$ such that there holds:

(i) $p_{Q}$ is the weak solution of the parabolic final time problem

$$
\begin{aligned}
-\frac{\partial p_{Q}}{\partial t}-A^{*} p_{Q} & =-\nabla \cdot\left(\nabla y_{Q}-d\right) \quad \text { in } Q, \\
p_{Q} & =0 \quad \text { on } \Sigma, \\
p_{Q}(\cdot, T) & =0 \quad \text { in } \Omega,
\end{aligned}
$$

(ii) The variational inequality

$$
\left(\int_{0}^{T}\left(\gamma_{\Sigma_{4}}\left(n_{\Sigma_{4}} \cdot R_{\Sigma_{4}}\left(p_{Q}\right)\right)\right) d t-g_{u}^{*}(u) p_{Q}(0)\right) \cdot(v-u) \geqslant 0, v \in U_{a d} .
$$


is satisfied, where $R_{\Sigma_{4}}(p)$ is given by

$R_{\Sigma_{4}}\left(p_{Q}\right)=\left(R_{\Sigma_{4}}^{(1)}\left(p_{Q}\right), R_{\Sigma_{4}}^{(2)}\left(p_{Q}\right)\right)^{T}, \quad R_{\Sigma_{4}}^{(k)}\left(p_{Q}\right):=S_{k}\left(\frac{1}{2} \sum_{\ell=1}^{2} \xi_{k \ell} S_{\ell} \frac{\partial p_{Q}}{\partial S_{\ell}}-r p_{Q}\right), 1 \leqslant k \leqslant 2$,

and $g_{u}^{*}(u) \in \mathscr{L}\left(L^{2}(\Omega), \mathbb{R}^{M}\right)$ stands for the adjoint of the Fréchet derivative of $g$ at $u \in U_{a d}$.

Proof. We introduce multipliers

$$
p=\left(p_{Q},\left(p_{\Sigma_{v}}\right)_{v=1}^{2}\right), q=\left(\left(q_{\Sigma_{v}}\right)_{v=1}^{4},\left(q_{K_{\max }, v}\right)_{v=1}^{2},\left(q_{K_{\min }, v}\right)_{v=1}^{2}, q_{0, \Omega},\left(q_{0, \Gamma_{v}}\right)_{v=1}^{2}\right) \text {, }
$$

such that

$$
\begin{aligned}
& p_{Q} \in W_{0}(0, T), p_{\Sigma_{v}} \in W_{v, 0}(0, T), 1 \leqslant v \leqslant 2 \\
& q_{\Sigma_{v}} \in L^{2}\left((0, T), H_{\omega}^{-1 / 2}\left(\Gamma_{v}\right)\right), 1 \leqslant v \leqslant 4, q_{K_{2}, v}, q_{K_{1}, v} \in \mathbb{R}, 1 \leqslant v \leqslant 2, \\
& q_{0, \Omega} \in L^{2}(\Omega), q_{0, \Gamma_{v}} \in L^{2}\left(\Gamma_{v}\right), 1 \leqslant v \leqslant 2 .
\end{aligned}
$$

We consider the Lagrangian

$$
\begin{aligned}
& L(y, u, p, q):=J\left(y_{Q}, u\right)+\int_{0}^{T}\left\langle\frac{\partial y_{Q}}{\partial t}-A(t) y_{Q}, p_{Q}\right\rangle d t+\sum_{v=1}^{2} \int_{0}^{T}\left\langle\frac{\partial y_{\Sigma_{v}}}{\partial t}-A_{v}(t) y_{\Sigma_{v}}, p_{\Sigma_{v}}\right\rangle d t \\
& +\sum_{v=1}^{4} \int_{0}^{T}\left\langle q_{\Sigma_{v}}, y_{\Sigma_{v}}-\gamma_{\Sigma_{v}}\left(y_{Q}\right)\right\rangle d t+\sum_{v=1}^{2} \int_{0}^{T}\left(q_{K_{\max , v}}\left(u_{M}-\gamma_{K_{\max }, v}\left(y_{\Sigma_{v}}\right)\right)-q_{K_{\min }, v} \gamma_{K_{\min }, v}\left(y_{\Sigma_{v}}\right)\right) \\
& +\left(y_{Q}(0)-g(u), q_{0, \Omega}\right)_{L^{2}(\Omega)}+\sum_{v=1}^{2}\left(y_{\Sigma_{v}}(0)-g(u), q_{0, \Gamma_{v}}\right)_{L^{2}\left(\Gamma_{v}\right)},
\end{aligned}
$$

where $\gamma_{\Sigma_{v}}\left(y_{Q}\right), 1 \leqslant v \leqslant 2$, is the trace of $y_{Q}$ on $\Sigma_{v}$ and

$$
\gamma_{K, v}\left(y_{\Sigma_{v}}\right)=y_{\Sigma_{v}}(K, \cdot), K \in\left\{K_{\min }, K_{\max }\right\}, 1 \leqslant v \leqslant 2
$$

Denoting by $A_{v}^{*}(t)$ the adjoint of $A_{v}(t), 1 \leqslant v \leqslant 2$, and introducing

$$
R_{K}\left(p_{v}\right):=\frac{1}{2} \sigma_{v}^{2} S_{K}^{2} \frac{\partial p_{\Sigma_{v}}}{\partial S_{v}}(K)-r S_{K} p_{\Sigma_{v}}(K), 1 \leqslant v \leqslant 2, K \in\left\{K_{\min }, K_{\max }\right\}
$$


integration by parts yields in time and an application of Green's formula results in

$$
\begin{aligned}
& L(y, u, p, q)= \\
& J(y, u)+\int_{0}^{T}\left\langle-\frac{\partial p_{Q}}{\partial t}-A^{*}(t) p_{Q}, y_{Q}\right\rangle d t+\sum_{v=1}^{2} \int_{0}^{T}\left\langle-\frac{\partial p_{\Sigma_{v}}}{\partial t}-A_{v}^{*}(t) p_{\Sigma_{v}}, y_{\Sigma_{v}}\right\rangle d t+ \\
& \sum_{v=1}^{4} \int_{0}^{T}\left(\left\langle\gamma_{\Sigma_{v}}\left(n_{\Sigma_{v}} \cdot R_{\Sigma_{v}}\left(p_{Q}\right)\right)-q_{\Sigma_{v}}, \gamma_{\Sigma_{v}}\left(y_{Q}\right)\right\rangle+\left\langle q_{\Sigma_{v}}, y_{\Sigma_{v}}\right\rangle\right) d t+ \\
& \left.\sum_{v=1}^{2} \int_{0}^{T}\left(R_{K_{\max }}\left(p_{\Sigma_{v}}\right)-q_{K_{\max , v}}\right) \gamma_{K_{\max }, v}\left(y_{\Sigma_{v}}\right)+q_{K_{\max }, v} u_{M}\right) d t- \\
& \left.\sum_{v=1}^{2} \int_{0}^{T}\left(R_{K_{\min }}\left(p_{\Sigma_{v}}\right)-q_{K_{\min , v},}\right) \gamma_{K_{\min }, v}\left(y_{\Sigma_{v}}\right)\right) d t+ \\
& \left(y_{Q}(0), q_{0, \Omega}-p_{Q}(0)\right)_{L^{2}(\Omega)}-\left(g(u), q_{0, \Omega}\right)_{L^{2}(\Omega)}+\left(y_{Q}(T), p_{Q}(T)\right)_{L^{2}(\Omega)}+ \\
& \sum_{v=1}^{2}\left(\left(y_{\Sigma_{v}}(0), q_{0, \Sigma_{v}}-p_{\Sigma_{v}}(0)\right)_{L^{2}\left(\Sigma_{v}\right)}-\left(g(u), q_{0, \Sigma_{v}}\right)_{L^{2}\left(\Sigma_{v}\right)}+\left(y_{\Sigma_{v}}(T), p_{\Sigma_{v}}(T)\right)_{L^{2}\left(\Sigma_{v}\right)}\right) .
\end{aligned}
$$

Here, $\left.R_{\Sigma_{v}}\left(p_{Q}\right)\right), 1 \leqslant v \leqslant 3$, is defined as in (4.3) with $\Sigma_{4}$ replaced by $\Sigma_{v}, 1 \leqslant v \leqslant 3$. In view of $J_{y}\left(y_{Q}, u\right)=-\nabla \cdot\left(\nabla y_{Q}-d\right)$, the optimality conditions

$$
L_{y}(y, u, p, q)=0 \quad \text { and } \quad L_{q}(y, u, p, q)=0
$$

reveal that

$$
\begin{aligned}
& q_{\Sigma_{v}}=\gamma_{\Sigma_{v}}\left(n_{\Sigma_{v}} \cdot R_{\Sigma_{v}}(p)\right), 1 \leqslant v \leqslant 4 \\
& q_{K_{\min }, v}=R_{K_{\min }}\left(p_{v}\right) \quad, \quad, q_{K_{\max }, v}=R_{K_{\max }}\left(p_{v}\right), 1 \leqslant v \leqslant 2, \\
& q_{0, \Omega}=\gamma_{0, \Omega}(p) \quad, \quad q_{0, \Sigma_{v}}=p_{v}(0), 1 \leqslant v \leqslant 2
\end{aligned}
$$

and

$$
\begin{aligned}
& \gamma_{\Sigma_{v}}\left(y_{Q}\right)=y_{\Sigma_{v}}, 1 \leqslant v \leqslant 4, \\
& \gamma_{K_{\min }, v}\left(y_{\Sigma_{v}}\right)=0, \quad \gamma_{K_{\max }, v}\left(y_{\Sigma_{v}}\right)=u_{M}, 1 \leqslant v \leqslant 2, \\
& y_{Q}(\cdot, 0)=g(u), y_{\Sigma_{v}}(\cdot, 0)=\left.g(u)\right|_{\Sigma_{v}}, 1 \leqslant v \leqslant 2 .
\end{aligned}
$$

Further, $p_{\Sigma_{v}}, 1 \leqslant v \leqslant 2$, is the weak solution of

$$
\begin{aligned}
-\frac{\partial p_{\Sigma_{v}}}{\partial t}-A_{v}^{*}(t) p_{\Sigma_{v}} & \left.=n_{\Sigma_{v}} \cdot R_{\Sigma_{v}}\left(p_{Q}\right)\right) \quad \text { in } \Sigma_{v}, \\
R_{K}\left(p_{\Sigma_{v}}\right) & =0, \quad K \in\left\{K_{\min }, K_{\max }\right\}, \\
p_{\Sigma_{v}}(\cdot, T) & =0 .
\end{aligned}
$$


Since $\left.n_{\Sigma_{v}} \cdot R_{\Sigma_{v}}\left(p_{Q}\right)\right)=0,1 \leqslant v \leqslant 2$, it follows that $p_{\Sigma_{v}}=0$ and hence $p_{\Sigma_{v}}=$ $\gamma_{\Sigma_{v}}\left(p_{Q}\right), 1 \leqslant v \leqslant 2$

Taking (4.5a)-(4.5c) and (4.6a)-(4.6c) into account, the optimality condition

$$
L_{p}(y, u, p, q)=0
$$

shows that $y_{Q}$ and $y_{\Sigma_{v}}, 1 \leqslant v \leqslant 2$, are the weak solutions of (3.5a)-(3.5c) and (3.6a)(3.6c). Finally, observing (2.8) and $y_{\Sigma_{4}}=u_{M}$ as well as the regularity results of Theorem 3.1, the optimality condition

$$
\left(L_{u}(y, u, p, q)\right) \cdot(v-u) \geqslant 0 \quad, \quad v \in U_{a d},
$$

gives rise to (4.2).

\section{Semi-discretization of the optimal control problem}

The parabolic problems (3.9a),(3.9b) and (3.10a),(3.10b) will be discretized in space by conforming P1 finite elements. To this end, we consider a shape-regular simplicial triangulation $\mathscr{T}_{h}(\Omega)$ of $\Omega$ which aligns with $\Gamma_{j}, 1 \leqslant j \leqslant 4$, so that this triangulation generates triangulations $\mathscr{T}_{h}\left(\Gamma_{j}\right)$ of $\Gamma_{j}, 1 \leqslant j \leqslant 4$, as well. We refer to $\mathscr{N}_{h}(D)$ and $\mathscr{E}_{h}(D), D \subseteq \bar{\Omega}$, as the sets of vertices in $D \subseteq \bar{\Omega}$. We denote by $h_{T}$ and $|T|$ the diameter and area of an element $T \in \mathscr{T}_{h}^{(m)}(\Omega)$. For $D \subset \bar{\Omega}$, we refer to $P_{k}(D), k \in \mathbb{N}_{0}$, as the linear spaces of polynomials of degree $\leqslant k$ on $D$.

We define $V_{h}$ as the finite element space of continuous P1 finite elements associated with the triangulation $\mathscr{T}_{h}(\Omega)$, i.e.,

$$
V_{h}:=\left\{v_{h} \in C(\bar{\Omega})\left|v_{h}\right|_{K} \in P_{1}(K), K \in \mathscr{T}_{h}(\Omega)\right\},
$$

and we set $V_{h, 0}:=V_{h} \cap C_{0}(\bar{\Omega})$. Likewise, we define $V_{h, v}, 1 \leqslant v \leqslant 2$, as the finite element spaces of continuous P1 finite elements associated with the triangulations $\mathscr{T}_{h}\left(\Gamma_{v}\right)$ attaining the values 0 at $S_{v}=K_{\min }$ and $u_{M}$ at $S_{v}=K_{\max }$, i.e.,

$$
V_{h, v}:=\left\{v_{h} \in C\left(\bar{\Gamma}_{v}\right)\left|v_{h}\right|_{K} \in P_{1}(K), K \in \mathscr{T}_{h}\left(\Gamma_{v}\right), v_{h}\left(K_{\min }\right)=0, v_{h}\left(K_{\max }\right)=u_{M}\right\},
$$

and we define $V_{h, v, 0}$ in the same way, but replacing $u_{M}$ with 0 .

The semi-discrete approximation of (3.9a),(3.9b) amounts to the computation of $y_{h, Q} \in C^{1}\left([0, T], V_{h}\right.$ with $\left.y_{h, Q}(\cdot, t)\right|_{\Gamma_{v}}=y_{h, \Gamma_{v}}(\cdot, t), 1 \leqslant v \leqslant 2$, and $\left.y_{h, Q}(\cdot, t)\right|_{\Gamma_{3}}=0,\left.y_{h, Q}(\cdot, t)\right|_{\Gamma_{4}}=$ $u_{M}$, such that

$$
\begin{aligned}
\left(\frac{d y_{h, Q}}{d t}, v_{h}\right)_{L^{2}(\Omega)}+a\left(t ; y_{h, Q}, v_{h}\right) & =0, v_{h} \in V_{h, 0}, \\
\left(y_{h, Q}(\cdot, 0), v_{h}\right)_{L^{2}(\Omega)} & =\left(g(u), v_{h}\right)_{L^{2}(\Omega)}, v_{h} \in V_{h} .
\end{aligned}
$$


On the other hand, for the semi-discrete approximation of (3.10a),(3.10b) we have to compute $y_{h, \Gamma_{v}} \in C^{1}\left([0, T], V_{h, v}\right), 1 \leqslant v \leqslant 2$, such that

$$
\begin{aligned}
\left(\frac{d y_{h, \Gamma_{v}}}{d t}, v_{h}\right)_{L^{2}\left(\Gamma_{v}\right)}+a\left(t ; y_{h, \Gamma_{v}}, v_{h}\right) & =0, v_{h} \in V_{h, v, 0}, \\
\left(y_{h, \Gamma_{v}}(\cdot, 0), v_{h}\right)_{L^{2}\left(\Gamma_{v}\right)} & =\left(g(u), v_{h}\right)_{L^{2}\left(\Gamma_{v}\right)}, v_{h} \in V_{h, v} .
\end{aligned}
$$

The semi-discrete optimal control problems reads: Find $\left(y_{h}, u\right)$, where $y_{h}=\left(y_{h, Q}, y_{h, \Gamma_{1}}, y_{h, \Gamma_{2}}\right)$ such that

$$
\begin{aligned}
& \inf _{y_{h}, u} J_{h}\left(y_{h}, u\right):=\frac{1}{2} \int_{0}^{T} \sum_{K \in \mathscr{T}_{h}(\Omega)} \| \nabla\left(y_{h, Q}(\cdot, t)-d \|_{L^{2}(K)}^{2} d t,\right. \\
& \text { subject to (5.4a),(5.4b),(5.3a),(5.3b) and (2.9). }
\end{aligned}
$$

The semi-discrete problem (5.5a), (5.5b) will be written in a more compact form as a control constrained optimal control problem for an initial-value problem associated with a system of first order linear ordinary differential equations. To this end, we set

$$
N_{\Omega}:=\operatorname{card}\left(\mathscr{N}_{h}(\Omega)\right), N_{\Gamma_{v}}:=\operatorname{card}\left(\mathscr{N}_{h}\left(\Gamma_{v}\right)\right), 1 \leqslant v \leqslant 4, N:=N_{\Omega}+N_{\Gamma_{1}}+N_{\Gamma_{2}},
$$

and denote by $\varphi_{h, \Omega}^{(i)}, 1 \leqslant i \leqslant N_{\Omega}, \varphi_{h, \Gamma v}^{(i)}, 1 \leqslant i \leqslant N_{\Gamma_{v}}$, the nodal basis functions associated with the nodal points in $\mathscr{N}_{h}(\Omega)$ and $\mathscr{N}_{h}\left(\Gamma_{v}\right), v \in\{1,2,4\}$. We introduce

$$
M=\left(\begin{array}{ccc}
M_{\Omega} & 0 & 0 \\
0 & M_{\Gamma_{1}} & 0 \\
0 & 0 & M_{\Gamma_{2}}
\end{array}\right) \quad, \quad A(t)=\left(\begin{array}{ccc}
A_{\Omega}(t) & A_{\Omega \Gamma_{1}}(t) & A_{\Omega \Gamma_{2}}(t) \\
0 & A_{\Gamma_{1}}(t) & 0 \\
0 & 0 & A_{\Gamma_{2}}(t)
\end{array}\right), t \in(0, T]
$$

as the block mass matrix $M \in \mathbb{R}^{N \times N}$ and the block stiffness matrix $A \in \mathbb{R}^{N \times N}$. Here, $M_{\Omega \Omega} \in \mathbb{R}^{N_{\Omega} \times N_{\Omega}}$ and $M_{\Gamma_{v} \Gamma_{v}}, 1 \leqslant v \leqslant 2$, stand for the matrices

$$
\begin{aligned}
\left(M_{\Omega}\right)_{i j} & :=\left(\varphi_{h, \Omega}^{(j)}, \varphi_{h, \Omega}^{(i)}\right)_{L^{2}(\Omega)}, 1 \leqslant i, j \leqslant N_{\Omega}, \\
\left(M_{\Gamma_{v}}\right)_{i j}: & :=\left(\varphi_{h, \Gamma_{v}}^{(j)}, \varphi_{h, \Gamma_{v}}^{(i)}\right)_{L^{2}\left(\Gamma_{v}\right)}, 1 \leqslant i, j \leqslant N_{\Gamma_{v}}, 1 \leqslant v \leqslant 2,
\end{aligned}
$$

whereas the matrices $A_{\Omega}(t) \in \mathbb{R}^{N_{\Omega} \times N_{\Omega}}, A_{\Omega \Gamma_{v}}(t) \in \mathbb{R}^{N_{\Omega} \times N_{\Gamma_{v}}}, A_{\Gamma_{v}}(t) \in \mathbb{R}^{N_{\Gamma_{v}} \times N_{\Gamma_{v}}}$ are given by

$$
\begin{aligned}
\left(A_{\Omega}\right)_{i j}: & =a\left(t ; \varphi_{h, \Omega}^{(j)}, \varphi_{h, \Omega}^{(i)}\right), 1 \leqslant i, j \leqslant N_{\Omega}, \\
\left(A_{\Omega \Gamma_{v}}\right)_{i j}: & =a\left(t ; \varphi_{h, \Gamma_{v}}^{(j)}, \varphi_{h, \Omega}^{(i)}\right), 1 \leqslant i \leqslant N_{\Omega}, 1 \leqslant j \leqslant N_{\Gamma_{v}}, 1 \leqslant v \leqslant 2, \\
\left(A_{\Gamma_{v}}\right)_{i j}: & =a\left(t ; \varphi_{h, \Gamma_{v}}^{(j)}, \varphi_{h, \Gamma_{v}}^{(i)}\right), 1 \leqslant i, j \leqslant N_{\Gamma_{v}}, 1 \leqslant v \leqslant 2 .
\end{aligned}
$$


We further introduce the input matrices

$$
\begin{aligned}
& B(t) \in \mathbb{R}^{N \times M}, B(t)=\left(0 B_{M}(t)\right), 0 \in \mathbb{R}^{N \times(M-1)}, \\
& B_{M}(t)=\left(B_{M, \Omega}(t), B_{M, \Gamma_{1}}(t), B_{M, \Gamma_{2}}(t)\right)^{T}, t \in(0, T], \\
& B_{M, \Omega}(t) \in \mathbb{R}^{N_{Q} \times 1},\left(B_{M, \Omega}(t)\right)_{i}:=-\sum_{j=1}^{N_{\Gamma_{4}}} a\left(t ; \varphi_{h, \Gamma_{4}}^{(j)}, \varphi_{h, \Omega}^{(i)}\right), 1 \leqslant i \leqslant N_{\Omega}, \\
& B_{M, \Gamma_{v}}(t) \in \mathbb{R}^{N_{\Gamma_{v}} \times 1},\left(B_{M, \Gamma_{v}}(t)\right)_{i}:=-a\left(t ; \varphi_{h, \Gamma_{4}}^{N_{\Gamma_{4}}^{(v)}}, \varphi_{h, \Gamma_{v}}^{(i)}\right), 1 \leqslant i \leqslant N_{\Gamma_{v}}, 1 \leqslant v \leqslant 2, \\
& \text { where } N_{\Gamma_{4}}^{(v)}:=(2-v)+(v-1) N_{\Gamma_{4}}, 1 \leqslant v \leqslant 2 \text {, and } \\
& G \in \mathbb{R}^{N \times M}, G=\left(G_{\Omega}, G_{\Gamma_{1}}, G_{\Gamma_{2}}\right)^{T}, G_{\Omega} \in \mathbb{R}^{N_{\Omega} \times M}, G_{\Gamma_{V}} \in \mathbb{R}^{N_{\Gamma_{v}} \times M}, \\
& \left(G_{\Omega}\right)_{i j}:=\int_{\Omega_{j}} g_{2}^{(j)}(S) \varphi_{h, \Omega}^{(i)}(S) d S+\int_{\Omega_{j+1}} g_{1}^{(j+1)}(S) \varphi_{h, \Omega}^{(i)}(S) d S, \\
& \left(G_{\Gamma_{v}}\right)_{i j}:=\int_{K_{j-1}}^{K_{j}} g_{2}^{(j)}\left(S_{V}\right) \varphi_{h, \Gamma_{v}}^{(i)}\left(S_{V}\right) d S_{v}+\int_{K_{j}}^{K_{j+1}} g_{1}^{(j+1)}\left(S_{V}\right) \varphi_{h, \Gamma_{v}}^{(i)}\left(S_{v}\right) d S_{v}, 1 \leqslant v \leqslant 2 . \\
& C_{\Omega} \in \mathbb{R}^{N_{\Omega} \times N_{\Omega}},\left(C_{\Omega}\right)_{i j}:=\sum_{T \in \mathscr{T}_{h}(\Omega)} \int_{T} \nabla \varphi_{h, \Omega}^{(j)} \cdot \nabla \varphi_{h, \Omega}^{(i)} d S, 1 \leqslant i, j \leqslant N_{\Omega}, \\
& D_{\Omega}^{(k)} \in \mathbb{R}^{N_{\Omega} \times N_{\Omega}},\left(D_{\Omega}^{(k)}\right)_{i j}:=\sum_{T \in \mathscr{T}_{h}(\Omega)} \int_{T} \frac{\partial \varphi_{h, \Omega}^{(j)}}{\partial S_{k}} \varphi_{h, \Omega}^{(i)} d S, 1 \leqslant i, j \leqslant N_{\Omega}, 1 \leqslant k \leqslant 2 .
\end{aligned}
$$

The semi-discrete optimal control problem reads as follows: Find $y \in C^{1}\left([0, T], \mathbb{R}^{N}\right), y=$ $\left(y_{Q}, y_{\Sigma_{1}}, y_{\Sigma_{1}}\right), u \in \mathscr{U}_{a d}$, such that

$$
\inf _{y, u} J(y, u):=\frac{1}{2} \int_{0}^{T}\left(y_{Q}^{T} C_{\Omega} y_{Q}-2 \sum_{k=1}^{2} d_{k}^{T} D_{\Omega}^{k} y_{Q}+\sum_{k=1}^{2} d_{k}^{T} M_{\Omega} d_{k}\right) d t,
$$

subject to

$$
\begin{aligned}
M \frac{d y}{d t}+A(t) y & =B u, t \in[0, T], \\
M y(0) & =G u .
\end{aligned}
$$


Theorem 5.1. The semi-discrete optimization problem (5.6a),(5.6b) admits a unique solution. If $y \in C^{1}\left([0, T], \mathbb{R}^{N}\right), u \in \mathscr{U}_{a d}$ is the optimal solution, there exists $p_{Q} \in C^{1}\left([0, T], \mathbb{R}^{N}\right)$ such that

$$
\begin{aligned}
M_{\Omega} \frac{d p_{Q}}{d t}-A(t)^{T} p_{Q} & =-C_{\Omega} y_{Q}+\frac{1}{2} \sum_{k=1}^{2}\left(D_{\Omega}^{(k)}\right)^{T} d_{k}, t \in[0, T], \\
M_{\Omega} p_{Q}(T) & =0,
\end{aligned}
$$

holds true and the variational inequality

$$
\left(-G^{T} p_{Q}(0)-\int_{0}^{T} B_{\Omega}(t)^{T} p_{Q} d t\right) \cdot(v-u) \geqslant 0, \quad v \in \mathscr{U}_{a d}
$$

is satisfied, where $B_{\Omega}(t)=\left(0 B_{M, \Omega}(t)\right), 0 \in \mathbb{R}^{(M-1) \times N_{\Omega}}$.

Proof. We introduce multipliers

$$
p_{Q} \in C^{1}\left([0, T], \mathbb{R}^{N}\right), \quad p_{\Sigma_{v}} \in C^{1}\left([0, T], \mathbb{R}^{N_{\Gamma_{v}}}, 1 \leqslant v \leqslant 2, \quad q_{0} \in \mathbb{R}^{N},\right.
$$

and consider the Lagrangian

$$
L\left(y, u, p, q_{0}\right):=J(y, u)+\int_{0}^{T} p \cdot\left(M \frac{d y}{d t}+A y-B u\right) d t+q_{0} \cdot(M y(0)-G u) .
$$

Integration by parts reveals

$$
\begin{aligned}
& L\left(y, u, p, q_{0}\right)=J(y, u)+\int_{0}^{T} y \cdot\left(-M \frac{d p}{d t}+A^{T} p\right) d t+-\int_{0}^{T} p \cdot B u d t \\
& +y(T) \cdot M p(T)-y(0) \cdot M p(0)+y(0) \cdot M q_{0}-q_{0} \cdot G u .
\end{aligned}
$$

The optimality condition $L_{y}\left(y, u, p, q_{0}\right)=0$ gives $q_{0}=p_{Q}(0)$ and shows that $p_{Q}$ satisfies (5.7a),(5.7b). Moreover, the optimality condition $L_{q_{0}}\left(y, u, p, q_{0}\right)=0$ yields $M y(0)=G u$ and together with $L_{p}\left(y, u, p, q_{0}\right)=0$ implies that $y$ satisfies (5.6a)(5.6c). Finally, $L_{u}\left(y, u, p, q_{0}\right)=0$ results in $(5.7 \mathrm{c})$.

\section{Fully discrete optimal control problem}

For the discretization in time of the semi-discrete optimal control problem (5.6a)(5.6c) we consider a partition

$$
0=: t_{0}<t_{1}<\cdots<t_{R}:=T / R, \quad R \in \mathbb{N},
$$


of the time interval $[0, T]$ with step lengths $\tau_{r}:=t_{r}-t_{r-1}, 1 \leqslant r \leqslant R$. We approximate the ODE (5.6b) by the backward Euler scheme, split the integral in the objective functional (5.6a) into the sum over the subintervals $\left(t_{r-1}, t_{r}\right)$ and use the quadrature formula $\int_{t_{r-1}}^{t_{r}} v d t \approx \tau_{r} v\left(t_{r}\right)$. Denoting by $y^{r}=\left(y_{Q}^{r}, y_{\Sigma_{1}}^{r}, y_{\Sigma_{1}}^{r}\right)^{T}$ approximations of $y=\left(y_{Q}, y_{\Sigma_{1}}, y_{\Sigma_{1}}\right)^{T}$ at $t_{r}, 0 \leqslant r \leqslant R$, and setting $y:=\left(y^{0}, \cdots, y^{R}\right)^{T}, y_{Q}:=$ $\left(y_{Q}^{0}, \cdots, y_{Q}^{R}\right)^{T}, y_{\Sigma_{v}}:=\left(y_{\Sigma_{v}}^{0}, \cdots, y_{\Sigma_{v}}^{R}\right)^{T}, 1 \leqslant v \leqslant 2$, the fully discrete optimal control problem reads: Find $(y, u) \in \mathbb{R}^{(R+1) N} \times U_{a d}$ such that

$$
\inf _{y, u} J(y, u):=\frac{1}{2} \sum_{r=1}^{R} \tau_{r}\left(\left(y_{Q}^{r}\right)^{T} C_{\Omega} y_{Q}^{r}-2 \sum_{k=1}^{2} d_{k}^{T} D_{\Omega}^{(k)} y_{Q}^{r}+\sum_{k=1}^{2} d_{k}^{T} M_{\Omega} d_{k}\right)
$$

subject to

$$
\begin{aligned}
M y^{r}+\tau_{r} A\left(t_{r}\right) y^{r} & =\tau_{r} B u+M y^{r-1}, 1 \leqslant r \leqslant R \\
M y^{0} & =G u .
\end{aligned}
$$

Theorem 6.1. The fully discrete optimization problem (6.1a)-(6.1c) admits a unique solution. If $y \in \mathbb{R}^{(R+1) N}, u \in U_{\text {ad }}$ is the optimal solution, there exists $p_{Q}=$ $\left(p_{Q}^{0}, \cdots, p_{Q}^{R}\right)^{T} \in \mathbb{R}^{(R+1) N_{\Omega}}$ such that

$$
\begin{aligned}
M_{\Omega} p_{Q}^{r-1}+\tau_{r} A_{\Omega}\left(t_{r-1}\right)^{T} p_{Q}^{r-1} & =M_{\Omega} p_{Q}^{r}+\tau_{r}\left(C_{\Omega} y_{Q}^{r}+\sum_{k=1}^{2}\left(D_{\Omega}^{(k)}\right)^{T} d_{k}\right), \\
M_{\Omega} p_{Q}^{R} & =0
\end{aligned}
$$

and

$$
\left(-G_{\Omega}^{T} p_{Q}^{0}-\sum_{r=0}^{R-1} \tau_{r+1} B\left(t_{r}\right)^{T} p_{Q}^{r}\right) \cdot(v-u) \geqslant 0 \quad, \quad v \in U_{a d}
$$

Proof. The proof is the discrete analogue of Theorem 5.1.

\section{Fully discrete optimal control problem}

We denote by $S: U_{a d} \rightarrow \mathbb{R}^{(R+1) N}$ the control-to-state map which assigns to an admissible control $u \in U_{a d}$ the solution $y \in \mathbb{R}^{(R+1) N}$ of the discrete state equation (6.1a),(6.1b). Then, the so-called control-reduced form of the fully discrete optimal control problem (6.1a)-(6.1c) reads

$$
\inf _{u \in U_{a d}} J^{r e d}(u), \quad J^{r e d}(u):=J(S(u), u) .
$$


It follows from Theorem 6.1 that the gradient of the control-reduced objective functional is given by

$$
\nabla J^{r e d}(u)=-G_{\Omega}^{T} p_{Q}^{0}-\sum_{r=0}^{R-1} \tau_{r+1} B_{\Omega}\left(t_{r}\right)^{T} p^{r}
$$

Denoting by $P_{U_{a d}}: \mathbb{R}^{M} \rightarrow U_{a d}$ the pointwise projection onto the admissible control set $U_{a d}$, i.e.,

$$
P_{U_{a d}}(w)=\left\{\begin{array}{c}
u_{i, \min }, \text { if } w_{i} \leqslant u_{i, \min } \\
w_{i}, \text { if } u_{i, \min } \leqslant w_{i} \leqslant u_{i, \max }, 1 \leqslant i \leqslant M, \\
u_{i, \max }, \text { if } w_{i} \geqslant u_{i, \max }
\end{array}\right.
$$

and given an initial control $u^{(0)} \in U_{a d}$, we solve (6.1a)-(6.1c) by the projected gradient method with Armijo line search (cf., e.g., $[17,25])$

$$
u^{(\ell+1)}=P_{U_{a d}}\left(u^{(\ell)}-\alpha_{\ell} \nabla J^{r e d}\left(u^{(\ell)}\right)\right), \ell \geqslant 0 .
$$

Here, $\alpha_{\ell}$ is the step length satisfying the Wolfe conditions

$$
\begin{aligned}
& J^{\text {red }}\left(u^{(\ell)}-\alpha_{\ell} \nabla J^{r e d}\left(u^{(\ell)}\right)\right) \leqslant J^{r e d}\left(u^{(\ell)}\right)-c_{1} \alpha_{\ell}\left\|\nabla J^{r e d}\left(u^{(\ell)}\right)\right\|^{2}, \\
& \nabla J^{r e d}\left(u^{(\ell)}\right)^{T} \nabla J^{r e d}\left(u^{(\ell)}-\alpha_{\ell} \nabla J^{r e d}\left(u^{(\ell)}\right)\right) \leqslant c_{2}\left\|\nabla J^{r e d}\left(u^{(\ell)}\right)\right\|^{2},
\end{aligned}
$$

with $0<c_{1} \ll c_{2}<1$. We note that (7.5a) is called the Armijo rule [3], whereas (7.5b) is referred to as the curvature condition. This leads to the following algorithm:

\section{Algorithm: Projected Gradient Method with Armijo Line Search}

Step 1: Choose a tolerance $T O L>0$ and an initial control $u^{(0)} \in U_{a d}$. Set $\ell:=0$.

Step 2: Compute $y^{(\ell+1)}=S\left(u^{(\ell)}\right) \in \mathbb{R}^{(R+1) N}$ and $p_{Q}^{(\ell+1)} \in \mathbb{R}^{(R+1) N_{\Omega}}$ as the solutions of (6.1b),(6.1c) and (6.2a),(??) (with $u$ in (6.1c) replaced with $\left.u^{(\ell)}\right)$. Update the control by computing $u^{(\ell+1)}$ according to (7.4) with steplength $\alpha_{\ell}$ chosen by means of $(7.5 \mathrm{a}),(7.5 \mathrm{~b})$.

Step 3: If the termination criterion $\left\|\nabla J^{r e d}\left(u^{(\ell)}\right)\right\|<T O L$ is satisfied, stop the algorithm and accept $\left(y^{(\ell+1)}, u^{(\ell+1)}, p_{Q}^{(\ell+1)}\right)$ as approximate solution of the fully discrete optimal control problem. Otherwise, set $\ell:=\ell+1$ and go to Step 2 .

\section{Numerical results}

We provide a documentation of computational results for the optimal control of European double barrier basket call options based on the numerical solution of the optimal control problem as outlined in section 5 and section 6 . We have considered the case of $M=5$ controls, i.e., $u=\left(u_{1}, \cdots, u_{5}\right)^{T}$ and various values of $d=\left(d_{1}, d_{2}\right)^{T}$ in the tracking-type objective functional of the optimal control problem. In the 
first example, we have used constant volatilities $\sigma_{1}, \sigma_{2}$ and interest rate $r$, whereas Example 2 deals with the case of variable data. Finally, in order to illustrate the benefits of the optimal control approach, Example 3 deals with a non-optimized European double barrier basket call option featuring a single cash-settlement at the upper barrier.

Example 1. Example 1. In our first example we have chosen $d=(0.2,0.2)^{T}$ in the objective functional. The complete data are given in Table 1 below.

\begin{tabular}{|c|c|r|}
\hline Parameter & Notation & Value \\
\hline$d$ & Desired Delta & $(0.2,0.2)^{T}$ \\
$M$ & Number of controls & 5 \\
$K_{\min }$ & Lower Barrier & 50 \\
$K_{\max }$ & Upper Barrier & 150 \\
$K$ & Strike & 100 \\
$T$ & Maturity & 1 \\
$r$ & Interest Rate & 0.05 \\
$\sigma_{1}$ & Volatility of asset 1 & 0.35 \\
$\sigma_{2}$ & Volatility of asset 2 & 0.20 \\
$\rho$ & Correlation between assets & -0.5 \\
$u_{i, \min }$ & Lower bound on the controls & 0.0 \\
$u_{i, \max }$ & Upper bound on the controls & 50.0 \\
\hline
\end{tabular}

Table 1. Example 1: Data of the optimal control problem
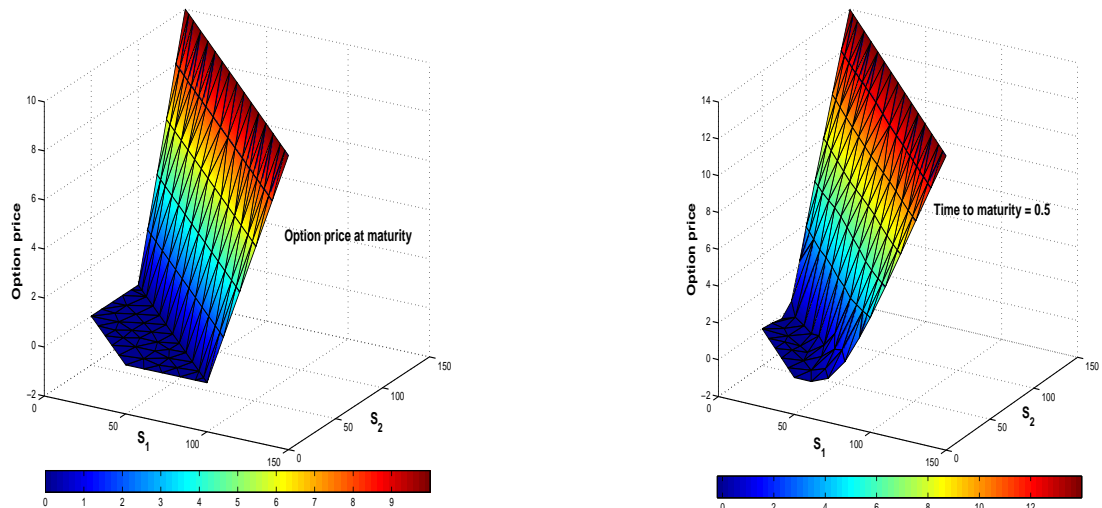

Figure 1. Example 1: Option price at maturity (left) and at time to maturity $t=0.5$ (right).

For discretization in space, we have chosen a simplicial triangulation of $\Omega$ with $h=5.0$ for both the state and the adjoint state and for discretization in time we 
have used a uniform time step of $\Delta t=0.01$. The projected gradient method with Armijo line search has been initialized with an initial control $u_{0}=(0,50,0,50,0)^{T}$ and has been stopped when the projected gradient became smaller than $T O L:=$ $1.0 E-06$. The iteration terminated after 48 iterations with the optimal control $u^{*}=$ $(2,4,6,8,10)^{T}$.

\begin{tabular}{|r|c|c||c|c|c|}
\hline$\ell$ & $J_{\text {red }}\left(u^{(\ell)}\right)$ & $\left\|\nabla J_{\text {red }}\left(u^{(\ell)}\right)\right\|$ & $\ell$ & $J_{\text {red }}\left(u^{(\ell)}\right)$ & $\left\|\nabla J_{\text {red }}\left(u^{(\ell)}\right)\right\|$ \\
\hline 1 & $2.5175 \mathrm{E}+03$ & $9.1334 \mathrm{E}+01$ & 10 & $3.9161 \mathrm{E}+01$ & $4.3928 \mathrm{E}+00$ \\
20 & $2.4413 \mathrm{E}+00$ & $6.4258 \mathrm{E}-02$ & 30 & $2.4400 \mathrm{E}+00$ & $4.3687 \mathrm{E}-03$ \\
40 & $2.4400 \mathrm{E}+00$ & $1.2411 \mathrm{E}-04$ & 48 & $2.4400 \mathrm{E}+00$ & $4.0830 \mathrm{E}-07$ \\
\hline
\end{tabular}

Table 2. Example 1 (Convergence history (maturity $t=T$ )): Number $\ell$ of projected gradient iteration, value $J_{\text {red }}\left(u^{(\ell)}\right)$ of the objective functional, and norm $\left\|\nabla J_{\text {red }}\left(u^{(\ell)}\right)\right\|$ of the gradient.

Table 2 contains a documentation of the convergence history of the projected gradient algorithm with Armijo line search. Here, $\ell$ stands for the iteration number, $J_{\text {red }}\left(u^{(\ell)}\right)$ is the corresponding value of the objective functional, and $\left\|\nabla J_{\text {red }}\left(u^{(\ell)}\right)\right\|$ refers to the norm of the gradient. As a termination criterion for the iteration, we have used $\left\|\nabla J_{\text {red }}\left(u^{(\ell)}\right)\right\|<T O L:=1.0 E-06$.

Example 2. The second example deals with the case of space-varying volatilities $\sigma_{1}, \sigma_{2}$, and time-varying interest rate $r$. The complete data are given in Table 3 below.

\begin{tabular}{|c|c|r|}
\hline Parameter & Notation & Value \\
\hline$d$ & Desired Delta & $(0.5,0.5)^{T}$ \\
$M$ & Number of controls & 5 \\
$K_{\min }$ & Lower Barrier & 50 \\
$K_{\max }$ & Upper Barrier & 150 \\
$K$ & Strike & 100 \\
$T$ & Maturity & 1 \\
$r$ & Interest Rate & $r(t)=0.02 \cdot t+0.08 \cdot(1-t)$ \\
$\sigma_{1}$ & Volatility of asset 1 & $\sigma_{1}(S, t)=0.75 \cdot\left(\left(S_{1}+S_{2}-100\right) / 50\right)^{2}$ \\
$\sigma_{2}$ & Volatility of asset 2 & $\sigma_{2}(S, t)=0.75 / 2 \cdot\left(\left(S_{1}+S_{2}-100\right) / 50\right)^{2}$ \\
$\rho$ & Correlation between assets & -0.5 \\
$u_{i, \min }$ & Lower bound on the controls & 0.0 \\
$u_{i, \max }$ & Upper bound on the controls & 50.0 \\
\hline
\end{tabular}

Table 3. Example 2: Data of the optimal control problem 
We have used the same discretizations and the same initial control as in the first example. As tolerance for the termination criterion we have used $T O L=10^{-6}$. The computed optimal control is $u^{*}=(17.50,18.98,25.07,29.40,35.77)^{T}$.
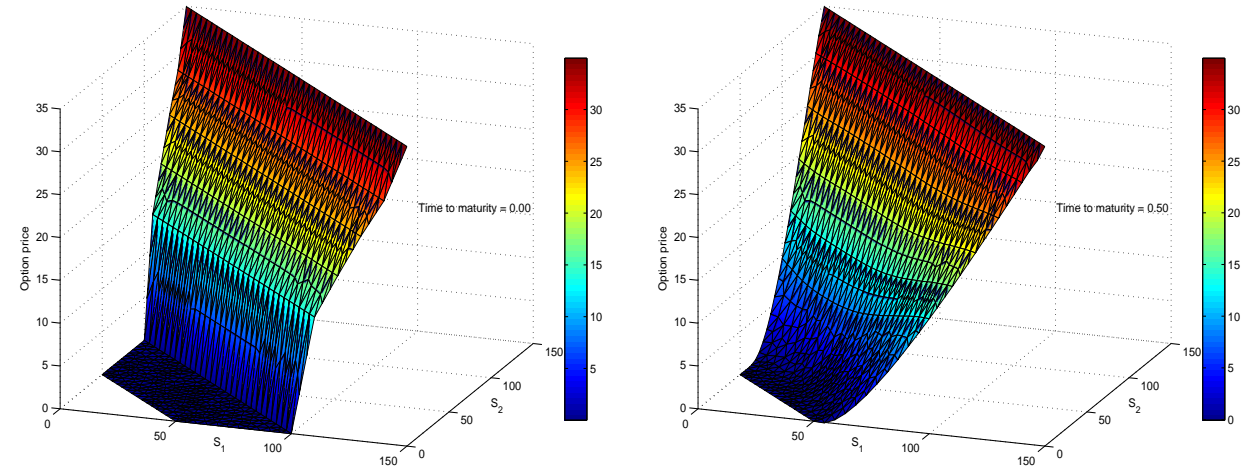

Figure 2. Example 2: Option price at maturity (left) and at time to maturity $t=0.5$ (right).

\begin{tabular}{|r|c|c||c|c|c|}
\hline$\ell$ & $J_{\text {red }}\left(u^{(\ell)}\right)$ & $\left\|\nabla J_{\text {red }}\left(u^{(\ell)}\right)\right\|$ & $\ell$ & $J_{\text {red }}\left(u^{(\ell)}\right)$ & $\left\|\nabla J_{\text {red }}\left(u^{(\ell)}\right)\right\|$ \\
\hline 1 & $1.5717 \mathrm{E}+03$ & $4.1822 \mathrm{E}+01$ & 10 & $1.0445 \mathrm{E}+02$ & $2.2505 \mathrm{E}+00$ \\
20 & $4.7608 \mathrm{E}+01$ & $2.2148 \mathrm{E}-02$ & 30 & $4.7412 \mathrm{E}+01$ & $3.2269 \mathrm{E}-04$ \\
40 & $4.7410 \mathrm{E}+01$ & $5.9259 \mathrm{E}-06$ & 46 & $4.7410 \mathrm{E}+01$ & $8.3484 \mathrm{E}-07$ \\
\hline
\end{tabular}

Table 4. Example 2 (Convergence history (maturity $t=T$ )): Number $\ell$ of the projected gradient iteration, value $J_{\text {red }}\left(u^{(\ell)}\right)$ of the objective functional, and norm $\left\|\nabla J_{\text {red }}\left(u^{(\ell)}\right)\right\|$ of the gradient.

The value of the value of the objective functional at optimality is significantly higher as in the first example due to the higher values of $d_{1}$ and $d_{2}$. On the other hand, the projected gradient method with Armijo line search performed similarly. Indeed, the termination criterion was reached after 46 iterations.

Example 3. In order to illustrate the benefits of optimized versus non-optimized European double barrier basket call options, we present the numerical results for a non-optimized call option with a single cash settlement of 10 at the upper barrier, but otherwise the same data as in the previous example.

In case the option is still in the money at maturity, its price corresponds to that of a plain vanilla European call option, as can be seen in Figure 3 (left). Otherwise, there are significant differences as displayed in Figure 3 (right). In fact, in comparison with European double barrier basket call options featuring optimized cash settlements and aiming at a constant Delta, the Delta is varying considerably and can even take negative values. 

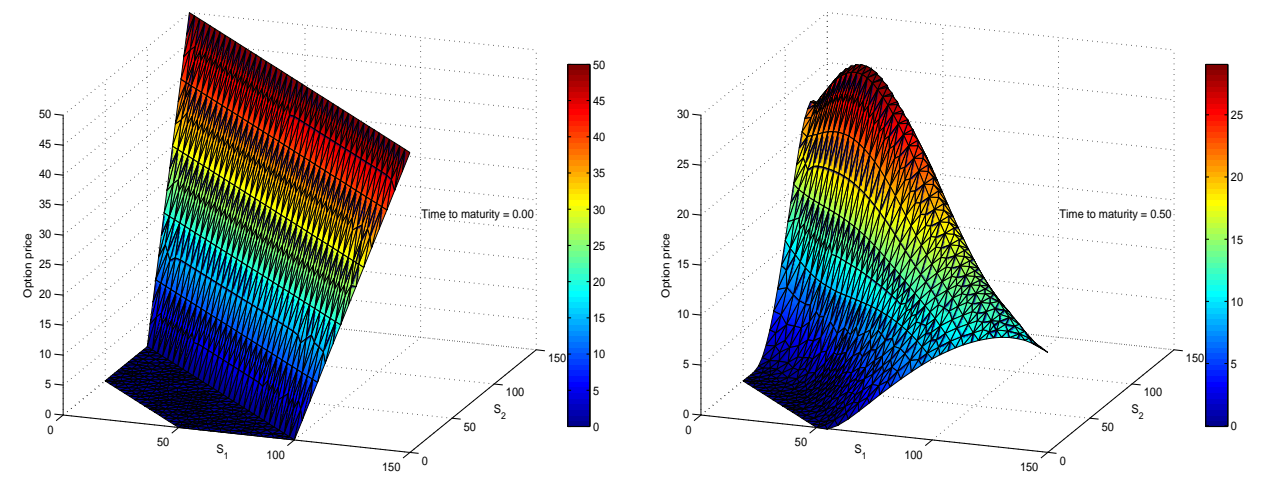

Figure 3. Example 3: Option price at maturity (left) and time to maturity $t=0.5$ (right).

\section{References}

1. Y. Achdou and O. Pironneau, Computational Methods for Option Pricing. SIAM, Philadelphia, 2005.

2. L. Andersen, J. Andreasen, and D. Eliezer, Static replication of barrier options: some general results. J. Comput. Finance 5, 1-25, 2002.

3. L. Armijo, Minimization of functions having Lipschitz continuous first partial derivatives. Pacific J. Math. 16 1-3, 1966.

4. M. Breitner and T. Burmester, Optimization of European double-barrier-options via optimal control of the Black-Scholes-equation. In: Operations Research Proceedings '01,(P. Chamoni et al.; eds.), Springer, Berlin-Heidelberg-New York, 2002.

5. M. Broadie and P. Glassermann, A continuity correction for discrete barrier options. Math. Finance 7, 325-349, 1997.

6. P. Carr, Two extensions to barrier option pricing. Appl. Math. Finance 2, 173-209, 1995.

7. P. Carr and A. Chou, Breaking barriers. Risk Magazine 10, 139-144, 1997.

8. P. Carr, K. Ellis, and V. Gupta, Static hedging of exotic options. J. Finance 53, 11651190, 1998.

9. T.H.E. Cheuk and T.C.F. Vorst, Complex barrier options. The Journal of Derivatives 4, 8-22, 1996.

10. D. Davydov and V. Linetsky, The valuation and hedging of barrier and lookback options under the CEV model. Management Science 47, 949-965, 2001.

11. E. Derman, D. Ergener, and I. Kani, Static Options Replication. J. of Derivatives 2, 78-95, 1995.

12. J. Dewynne, S. Howison, and P. Wilmott, The Mathematics of Financial Derivatives. 
Cambridge University Press, Cambridge, 1995.

13. A.V. Fursikov, Optimal Control of Distributed Systems: Theory and Applications. American Math. Society, Providence, 1999.

14. A.M. Giese and J.H. Maruhn, Cost-optimal static super-replication of barrier options - an optimization approach. J. Comput. Finance 10, 71-97, 2007.

15. R. Heynen and H. Kat, Crossing Barriers. Risk Magazine 7, 46-51, 1994.

16. M. Jeanblanc, M. Yor, and M. Chesney, Mathematical Models for Financial Markets. Springer, Berlin-Heidelberg-New York, 2003.

17. C.T. Kelley, Iterative Methods for Optimization. SIAM, Philadelphia, 1999.

18. Y.-K. Kwok, L. Wu, and H. Yu, Pricing multi-asset options with an external barrier. Int. J. Theoretical Appl. Finance 1, 523-541, 1998.

19. C.F. Lo, P.H. Yuen, and C.H. Hui, A simple approach for pricing barrier options with time-dependent parameters. Quantitative Finance 3, 98-107, 2003.

20. J.H. Maruhn, Duality in static hedging of barrier options. Optimization 58, 319-333, 2009.

21. J.H. Maruhn and E.W. Sachs, Robust static hedging of barrier options in stochastic volatility models. Mathematical Methods of Operation Research 70, 405-433, 2009.

22. R. Merton, The theory of rational option pricing. Bell Journal of Economics and Management Science 4, 141-183, 1973.

23. M. Nalholm and R. Poulsen, Static hedging of barrier options under general asset dynamics: unification and application. J. Derivatives 13, 46-60, 2006.

24. I. Nelken, Pricing, Hedging, and Trading Exotic Options. McGraw-Hill, New York, 1999.

25. J. Nocedal and S.J. Wright, Numerical Optimization. 2nd Edition. Springer, BerlinHeidelberg-New York, 1999.

26. R. Poulsen, Barrier options and their static hedges: simple derivations and extensions. Quant. Finance 6, 327-335, 2006.

27. E. Reiner and M. Rubinstein, Breaking down the barriers. Risk Magazine 4, 28-35, 1991.

28. M. Renardy and R.C. Rogers, An Introduction to Partial Differential Equations. Springer, Berlin-Heidelberg-New York, 1993.

29. P. Ritchken, On pricing barrier options. In: Currency derivatives: pricing theory, exotic options, and hedging applications (D.F. DeRosa, Ed.), pp. 275-289, J. Wiley \& Sons, New York, 1998.

30. A. Sbuelz, Hedging double barriers with singles. Int. J. Theoretical and Applied Finance 8, 393-406, 2005.

31. J. Topper, Finite element modeling of exotic options. Discussion Paper No. 216, Arthur Andersen Risk Management, Eschborn, 1998.

32. F. Tröltzsch, Optimal Control of Partial Differential Equations. Theory, Methods, and Applications. American Mathematical Society, Providence, 2010.

33. H.Y. Wong and Y.-K. Kwok, Multi-asset barrier options and occupation time deriva- 
tives. Appl. Math. Finance 10, 245-266, 2003.

34. P.G. Zhang, Exotic Options. World Scientific, Singapore, 1997. 